\title{
Wastewater monitoring outperforms case numbers as a tool to track COVID-19 incidence dynamics when test positivity rates are high
}

\author{
Xavier Fernandez-Cassi ${ }^{a}$, Andreas Scheidegger ${ }^{b}$, Carola Bänziger ${ }^{b}$, Federica Cariti ${ }^{a}$, \\ Alex Tuñas Corzon a, Pravin Ganesanandamoorthy ${ }^{b}$, Joseph C. Lemaitre ${ }^{c}$, Christoph Ort ${ }^{b}$, \\ Timothy R. Julian a,d,e, Tamar Kohn ${ }^{\text {a,* }}$ \\ a Laboratory of Environmental Chemistry, School of Architecture, Civil and Environmental Engineering, École Polytechnique Fédérale de Lausanne (EPFL), \\ CH-1015 Lausanne, Switzerland \\ b Swiss Federal Institute of Aquatic Science and Technology (Eawag), 8600 Dübendorf, Switzerland \\ ${ }^{c}$ Laboratory of Ecohydrology, School of Architecture, Civil and Environmental Engineering, École Polytechnique Fédérale de Lausanne (EPFL), CH-1015 \\ Lausanne, Switzerland \\ d Swiss Tropical and Public Health Institute, CH-4051 Basel, Switzerland \\ e University of Basel, CH-4055 Basel, Switzerland
}

\section{A R T I C L E I N F O}

\section{Article history:}

Received 27 March 2021

Revised 10 May 2021

Accepted 11 May 2021

Available online 17 May 2021

\section{Keywords:}

Sewage

New infections

Compartmental model

Shedding load distribution

SARS-CoV-2

Disease dynamics

\begin{abstract}
A B S T R A C T
Wastewater-based epidemiology (WBE) has been shown to coincide with, or anticipate, confirmed COVID19 case numbers. During periods with high test positivity rates, however, case numbers may be underreported, whereas wastewater does not suffer from this limitation. Here we investigated how the dynamics of new COVID-19 infections estimated based on wastewater monitoring or confirmed cases compare to true COVID-19 incidence dynamics. We focused on the first pandemic wave in Switzerland (February to April, 2020), when test positivity ranged up to 26\%. SARS-CoV-2 RNA loads were determined 2-4 times per week in three Swiss wastewater treatment plants (Lugano, Lausanne and Zurich). Wastewater and case data were combined with a shedding load distribution and an infection-to-case confirmation delay distribution, respectively, to estimate infection incidence dynamics. Finally, the estimates were compared to reference incidence dynamics determined by a validated compartmental model. Incidence dynamics estimated based on wastewater data were found to better track the timing and shape of the reference infection peak compared to estimates based on confirmed cases. In contrast, case confirmations provided a better estimate of the subsequent decline in infections. Under a regime of high-test positivity rates, WBE thus provides critical information that is complementary to clinical data to monitor the pandemic trajectory.
\end{abstract}

(C) 2021 The Author(s). Published by Elsevier Ltd. This is an open access article under the CC BY-NC-ND license (http://creativecommons.org/licenses/by-nc-nd/4.0/)

\section{Introduction}

Wastewater-based epidemiology (WBE), a form of environmental surveillance of infectious diseases, has long been suggested as a sensitive tool to monitor pathogen circulation in a population (Fernandez-Cassi et al., 2018; Hovi et al., 2012; Sinclair et al., 2008). Many pathogens, both enteric and otherwise, are excreted from infected individuals into the sewage system via feces, saliva or other bodily fluids (Sinclair et al., 2008). The principle underlying WBE is that the pathogen concentrations or loads in sewage

\footnotetext{
* Corresponding author.

E-mail address: tamar.kohn@epfl.ch (T. Kohn).
}

are proportional to the number of infected individuals among the population contributing to the sewage, and can thus inform on the presence and trajectory of a disease outbreak. For example, norovirus concentrations in sewage were found to closely track the dynamics gastroenteritis cases over several years in Japan (Kazama et al., 2017). WBE can inform not only on the presence and dynamics of a pathogen but may also capture the emergence of new strains or variants before they become widespread in a population (Bisseux et al., 2020; Kazama et al., 2017).

WBE has received renewed attention during the COVID-19 pandemic, when it was recognized that SARS-CoV-2 RNA is excreted in feces (Y. Wu et al., 2020) and can be detected in wastewater (Ahmed et al., 2020; Medema et al., 2020; Randazzo et al., 2020) and sludge (Graham et al., 2021; Peccia et al., 2020). Several 
studies have shown that the dynamics of SARS-CoV-2 RNA in raw wastewater or sludge coincide with, or even anticipate, the dynamics of confirmed cases (Graham et al., 2021; Medema et al., 2020; Peccia et al., 2020). In addition, WBE was able to capture the introduction and spread of SARS-CoV-2 variants of concern (Jahn et al., 2021), and identify mutations that were not captured in clinical samples (Crits-Christoph et al., 2021). WBE may thus serve as a useful tool to support COVID-19 monitoring, and WBE data have already been integrated into multiple national or local COVID-19 dashboards.

While WBE will never replace case reporting, it can be used to strengthen the understanding of infectious disease dynamics as it holds important benefits over clinical tests. Specifically, WBE captures both symptomatic and asymptomatic virus shedders; WBE data are not affected by testing capacity, strategy or compliance; and WBE allows the monitoring of a large population with few samples. The advantages of WBE over clinical testing are particularly important when test capacity is exceeded and hence may be insufficient to accurately capture case numbers. According to the $\mathrm{WHO}$, the test positivity rate should remain $<5 \%$ to confidently track disease dynamics (World Health Organization, 2020). Under regimes with a positivity rate $>5 \%$, WBE may thus better reflect true disease dynamics than clinical case numbers.

In this study we evaluated the use of wastewater monitoring as a tool to track COVID-19 dynamics. We hypothesized that under high test positivity rates, wastewater provides an improved estimate of the dynamics of new infections (incidence dynamics) compared to confirmed case numbers. We focused on the first wave of the COVID-19 pandemic in Switzerland, which lasted from late February to April 2020. The test positivity rate during this period ranged up to $26 \%$ (Figure S1). Wastewater was monitored 2-4 times per week in two locations (Lugano, Lausanne) that were strongly affected, and one location (Zurich) that experienced a milder wave. We did not directly evaluate SARS-CoV-2 RNA loads measured in wastewater against the number of confirmed cases. Instead, we use these metrics to estimate the incidence dynamics over time. This allowed us to compare both the wastewater- and the case number-derived incidence dynamics to reference incidence dynamics determined retrospectively by a compartmental (Susceptible-Exposed-Infected-Recovered; SEIR) model and consistent with seroprevalence studies conducted in the region (Lemaitre et al., 2020).

\section{Materials and methods}

\subsection{Experimental approach}

We determined the concentration and daily loads of SARS-CoV2 RNA in longitudinal samples of raw wastewater collected from three Swiss wastewater treatment plants (WWTPs). In each sample, we analyzed two SARS-CoV-2 gene targets (N1 and N2). In addition, we determined virus recovery by means of an externally added viral surrogate of SARS-CoV-2. Finally, we monitored the fecal strength in each sample via the analysis of pepper mild mottle virus (PMMoV), a plant virus that occurs in wastewater at high and constant concentrations (Kitajima et al., 2014; Symonds et al., 2018).

\subsection{Sample collection and storage}

24-h composite influent samples were collected 2-4 times per week between February 26 and April 30, 2020 from three Swiss WWTPs: Lausanne (STEP de Vidy; population connected: $240^{\prime} 000$; 25 samples), Lugano (CDA Bioggio; population connected: 125'000; 31 samples); and Zurich (ARA Werdhölzli: population connected: $450^{\prime} 000 ; 22$ samples). After collection, the wastewater samples were stored at $-20 \stackrel{\circ}{ } \mathrm{C}$ for up to 5 months.

\subsection{Preparation of viral surrogate stock solutions}

Three enveloped viruses were assessed as external recovery controls, namely Murine Hepatitis Virus (MHV, Coronaviridae, betacoronavirus), Pseudomonas virus $\Phi 6$ (Cystoviridae, cystovirus) and Murine Sendai virus (Paramyxoviridae, respirovirus). Murine Hepatitis Virus strain MHV-A59 (kindly donated by Volker Thiel, University of Bern) was propagated in delayed brain tumor (DBT) cells (kindly donated by Krista Rule Wigginton, University of Michigan) as described elsewhere (Leibowitz et al., 2011). Five days post-infection the viral particles were released from infected cells by three cycles of freezing/thawing. Cell supernatants were centrifuged at $3000 \times \mathrm{g}$ to pellet down cell debris and the supernatant was clarified through a $0.22 \mu \mathrm{m}$ filter. The resulting stock solution had a concentration of $7.8 \times 10^{9}$ genome copies $(\mathrm{gc}) / \mathrm{ml}$. Bacteriophage $\Phi 6$ (DSMZ no 21518, strain HER 102, Braunschweig, Germany) was propagated in P. syringae (DSMZ no 21482, strain HER1102) according to the provider's instructions. After propagation, bacterial cultures were centrifuged at $8000 \times \mathrm{g}$ for $10 \mathrm{~min}$ and cell debris was removed by passing the supernatant through a $0.22 \mu \mathrm{m}$ filter. The final stock solution had a concentration of $8.0 \times 10^{8} \mathrm{gc} / \mathrm{ml}$. Finally, Sendai virus propagated in embryonated eggs was kindly donated by Dominique Garcin (University of Geneva) and was used without further treatment. These solutions had a concentration of $1.3 \times 10^{9} \mathrm{gc} / \mathrm{ml}$.

\subsection{Sample concentration and nucleic acid extraction}

Samples from Lugano and Lausanne were processed at EPFL, and samples from Zurich were processed at Eawag. Prior to processing, samples were thawed at room temperature. For each sample, two replicate aliquots of $50 \mathrm{ml}$ wastewater were processed. The $50 \mathrm{ml}$ aliquots were spiked with MHV (Lausanne or Lugano) or Sendai virus (Zurich) at a concentration of approximately $1 \times 10^{6}$ $\mathrm{gc} / 50 \mathrm{ml}$, and were stirred for 20 minutes to ensure the homogenization of the sample. Then they were pre-filtered using $2 \mu \mathrm{m}$ glass fiber pre-filters (cat no AP2007500, Merck Millipore, Burlington, MA, USA) placed on the top of $0.22 \mu \mathrm{m}$ SteriCup filters (cat no SCGVU02RE, Merck Millipore). After filtration, the filter units were rinsed with $10 \mathrm{ml}$ of ultrapure water to ensure that no wastewater was retained in the dead volume. The filtrates (approximately $60 \mathrm{ml}$ ) were transferred to a centrifugal filter unit with a size cutoff of 100 kDa (Centricon Plus-70; cat no UFC701008, Merck Millipore), and were centrifuged for $30 \mathrm{~min}$ at $3000 \times \mathrm{g}$. To collect the concentrate, the centrifugal filter was inverted and centrifuged for $3 \mathrm{~min}$ at $1000 \times \mathrm{g}$. The resulting viral concentrate volume ranged from 180 to $300 \mu \mathrm{L}$.

Viral concentrates were extracted in their entirety using the Qiagen RNA Viral Mini Kit (cat no 22906, Qiagen, Valencia, CA, USA) following the manufacturer's protocol for higher volumes. Nucleic acids were eluted using $80 \mu \mathrm{l}$ of AVE buffer. For each processed batch of samples, a negative extraction control using water was included. The extracted nucleic acids were passed through a Zymo OneStep PCR Inhibitor Removal column (cat no D6030, Zymo Research, Irvine, CA, USA) to remove PCR inhibitors following the protocol provided by the manufacturer.

In addition to the longitudinal samples, seven composite wastewater samples were collected in Lausanne to test the recovery of different SARS-CoV-2 surrogates. These samples were spiked with MHV, Sendai virus and $\Phi 6$ at a concentration of approximately $10^{6} \mathrm{gc} / 50 \mathrm{ml}$ each. Samples were then concentrated and nucleic acids were extracted as described above. 


\subsection{Quantification of SARS-CoV-2 N1 and N2 genes, viral surrogates and $P M M o V$ by $R T-q P C R$}

All RNA extracts of the longitudinal samples were analyzed by RT-qPCR for four viral targets: The N1 and N2 gene targets of SARSCoV-2, the surrogate virus and PMMoV. All N1, N2 and MHV analyses, as well as PMMoV analyses for Lugano and Lausanne were performed at EPFL. PMMoV and Sendai virus analyses for Zurich were beyond the average Cq measured at a given site. All inhibition tests were conducted at EPFL.

\subsection{Recovery}

Recovery was calculated as the ratio of surrogate virus recovered after sample processing and the virus originally spiked into $50 \mathrm{ml}$ of unfiltered wastewater (Eq. 1):

$$
\text { Recovery }=\frac{\text { virus measured per } \mu \text { lRNAextract } \times \text { RNAextractionvolume }(80 \mu \mathrm{l})}{\text { virus spiked into } 50 \mathrm{ml} \text { of wastewater }}
$$

$\overline{\text { performed at Eawag. The samples to test surrogate virus recovery }}$ were analyzed at EPFL for three viral targets: MHV, Sendai virus and $\Phi 6$. To detect the presence of SARS-CoV-2 RNA, the CDC N1 and N2 assays were used (Lu et al., 2020). PMMoV and MHV were analyzed by previously reported assays (Besselsen et al., 2002; Haramoto et al., 2013; Zhang et al., 2006). The design for primers and probes for $\Phi 6$ were adapted from Gendron et al. (2010) according to the suggestion of Heather Bischel (University of California, Davis). For Sendai virus, primers and probes were designed for the purpose of this project. A summary of all primers and probes and the RT-qPCR protocols is given in the Supporting Information (Table S1)

To calibrate the different RT-qPCR assays, standard curves for each viral target were generated using either double-stranded DNA gblocks gene fragments (viral surrogates and PMMoV), or a 2019nCoV_N positive control plasmid (cat no 10006625 , SARS-CoV-2 N1 and N2). Both gblocks and plasmids were purchased from Integrated DNA Technologies (Coralville, IA, USA).

RT-qPCR amplifications were performed in $25 \mu \mathrm{l}$ reactions using RNA UltraSense ${ }^{\mathrm{TM}}$ One-Step Quantitative RT-PCR System (cat no 11732-927, Invitrogen, Carlsbad, CA, USA), amended with $4 \mu \mathrm{l}$ of bovine serum albumin ( $2 \mathrm{mg} / \mathrm{ml}$; cat. no 10711454001, SigmaAldrich, Buchs, Switzerland) on a Mic qPCR Cycler (Bio Molecular Systems, Upper Coomera, Queensland, Australia). In each RT-qPCR reaction, $5 \mu \mathrm{l}$ of RNA extract or calibration standard were used. For PMMoV, the RNA extract was diluted 1:10 prior to RT-qPCR analysis. All RT-qPCR runs included no-template controls and negative extraction controls to monitor for contamination during the extraction and amplification process. The preparation of PCR mastermix and standards, as well as sample loading were performed in separate locations to avoid contamination. Cq determination was performed using the micPCR software (v2; Bio Molecular Systems).

RT-qPCR limits of detection (LOD) were determined as the lowest concentration (N1, N2 and MHV) or the lowest standard (PMMoV, Sendai, $\Phi 6$ ) with a $95 \%$ or greater detection probability. The limit of detection (LOD) for each gene target were determined from pooled standard curves $(n \geq 3)$ in $R$ using the Generic qPCR Limit of Detection (LOD) / Limit of Quantification (LOQ) calculator (Merkes et al., 2019). Samples with a measurable RT-qPCR signal < LOD were assigned the concentration of the LOD of the respective assay. Samples which yielded no detectable RT-qPCR signal were set to the theoretical minimal LOD ( $3 \mathrm{gc} /$ reaction) (Ståhlberg and Kubista, 2014).

\section{6. $R T-q P C R$ inhibition}

To check for inhibition during RT-qPCR reactions, $4 \mu \mathrm{l}$ of each Zymo-treated RNA extract were amended with $1 \mu$ of a synthetic SARS-CoV-2 RNA reference material (cat. no EURM-019, European Commission, Joint Research Center, Geel, Belgium) at a concentration of approximately $10^{5} \mathrm{gc} / \mu \mathrm{L}$. RNA extracts were analyzed for the SARS-CoV-2 N1 target by the RT-qPCR protocol described above, and the resulting Cq values were compared between samples. Samples were considered inhibited when Cq was $>1.5$ cycles

\subsection{Determination of RNA loads}

Genome copies $(\mathrm{gc})$ per reaction were converted to units of load (gc/day) by determining the gc concentration per liter of wastewater and multiplication by the wastewater flow rate of corresponding day according to Equation 2:

Load $(\mathrm{gc} /$ day $)=\frac{\mathrm{C}_{\mathrm{PCR}} \times \mathrm{V}_{\text {extract }} / \mathrm{V}_{\mathrm{PCR}}}{\mathrm{V}_{\text {sample }} \times \mathrm{Q}}$

Where $\mathrm{C}_{\mathrm{PCR}}$ is the template concentration ( $\mathrm{gc} /$ reaction) determined by RT-qPCR, $V_{\text {extract }}$ is the total volume of RNA extract ( 80 $\mu \mathrm{L}), V_{P C R}$ is the volume of extract analyzed by RT-qPCR $(5 \mu \mathrm{L})$, $V_{\text {sample }}$ is the volume of the wastewater sample $(0.05 \mathrm{~L})$, and $Q$ is the wastewater flow rate on a given sampling day measured and provided by the WWTPs included in this study (L/day).

\subsection{Storage test}

To determine if storage at $-20{ }^{\circ} \mathrm{C}$ had a detrimental effect on SARS-CoV-2 RNA concentrations in wastewater, a control experiment was conducted. A batch of wastewater influent from Lausanne was collected and stored for a month in four different conditions: 1) unprocessed wastewater at $4{ }^{\circ} \mathrm{C} ; 2$ ) unprocessed wastewater at $-20{ }^{\circ} \mathrm{C} ; 3$ ) concentrated wastewater (after ultrafiltration) at $-20{ }^{\circ} \mathrm{C}$; and 4) Zymo-treated RNA extract at $-20{ }^{\circ} \mathrm{C}$. All tests were conducted in duplicate. After one month, all samples were fully processed and immediately analyzed for the N1 gene target by RTqPCR as described above.

\subsection{Epidemiological data}

Confirmed case numbers for each WWTP catchment and test positivity rates were kindly provided by the Swiss Federal Office of Public Health.

\subsection{Incidence estimates}

Reference infection numbers were determined by an SEIR model described previously (Lemaitre et al., 2020). This model is based on cantonal hospitalization data, intensive care unit visits and deaths, but not case numbers. This is to avoid any influence from changes in test strategies and test capacity over the time period considered. The modeled incidence includes both symptomatic and asymptomatic new infections. The model was validated against a seroprevalence study conducted in the region, and we therefore consider it herein as the reference incidence.

Incidence was additionally estimated based on longitudinal data of SARS-CoV-2 loads in wastewater and based on confirmed cases. Both these metrics measured at time $t$ reflect an aggregate of infections that occurred over a time span preceding the measurement. A deconvolution of these aggregated quantities allows for the reconstruction of daily infections. This requires an assumption of the extent by which previous infections influence the aggregated 


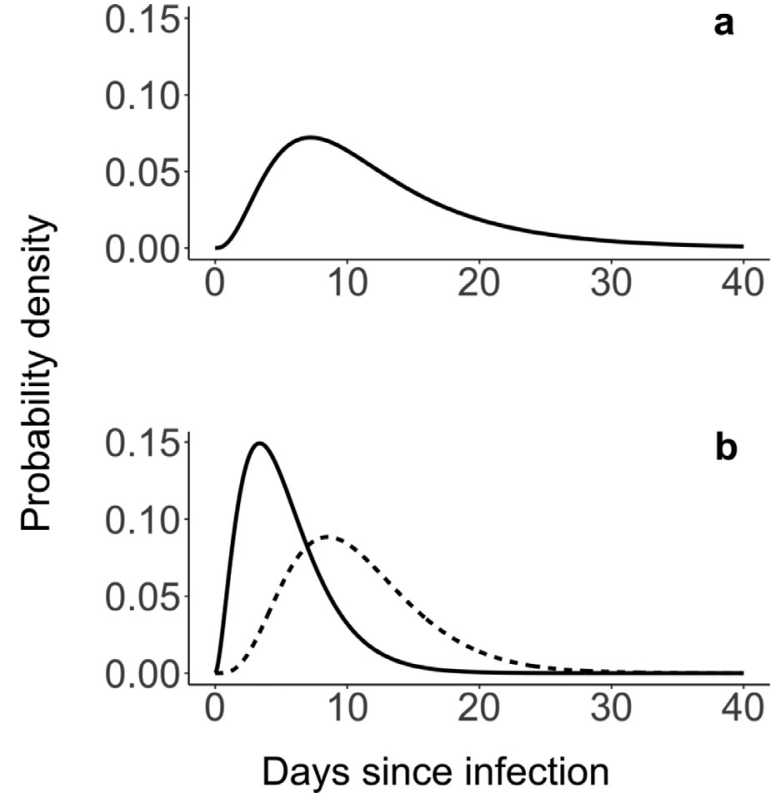

Fig. 1. Delay distributions for virus shedding and case confirmation. a) Shedding load distribution, based on the delay distribution from infection to symptom onset by Linton et al. (2020), combined with the gastrointestinal viral load dynamics according to Benefield et al. (2020) b) Delay distribution from infection to symptom onset according to Linton et al. (2020) (solid line), and combined with an additional delay from symptom onset to case confirmation based on Bi et al. (2020) (dashed line).

quantities. This assumption is expressed by time delay-dependent weight $\omega(\tau)$, where $\tau$ is the time (days) since infection. The measured aggregated quantity $A$ at time $t$ can be approximated by a weighted sum of all infections $I$ that occurred up to time $t$ :

$A_{t} \approx \sum_{\tau=0}^{\infty} \omega(r) l_{t-\tau}$

The infections $I$ over a time range of interest can be estimated via non-negative least squares regression. As fast fluctuations in the number of daily infections seems unreasonable, an additional constraint was added to enforce smoothness comparable to the infection numbers of the SEIR model (see Supporting Information).

To obtain the wastewater-derived incidence, the weights of the deconvolution, $\omega(\tau)$, are given by the shedding load profile (SLP) that describes the average amount of virus shed by a patient $\tau$ days after infection. The SLP can be decomposed into the relative shedding load probability distribution (SLD) and the absolute viral load shed during the course of the disease (L) (Eq. 4):

$\operatorname{SLP}(\tau)=L \times \operatorname{SLD}(\tau)$

The SLD was constructed by combining the gastrointestinal viral load as a function of time after symptom onset with the time between infection and symptoms. Virus shedding was modelled based on data reviewed by Benefield et al. (2020), and could be well described by a gamma distribution with a mean of 6.73 days and a standard deviation (sd) of 6.98 days. The time between infection and symptoms was also modeled by a gamma distribution based on Linton et al. (2020) (mean $=5.3$ days, sd $=3.2$ days). The convolution of these two distributions was used as the SLD (shown in Fig. 1 a) with a mean $=11.73$ days and an $s d=7.68$ days.

If $L$ is known with small uncertainty, the absolute number of infections can be estimated. However, although the different SLDs have a comparable shape across literature, the loads $L$ are highly variable (Han et al., 2020; Liu et al., 2020). Therefore, we applied the SLD, which still yields an estimate that is proportional to that obtained by using the correct but unknown SLP.
For the case number-derived incidence, $\omega(\tau)$ was defined by the distribution combining the delays from infection to symptom onset (gamma mean $=5.3$ days; sd $=3.2$ days), and from symptom onset to case confirmation (gamma mean $=5.5$ days, sd $=3.8$ days; Bi et al., 2020). The resulting delay distribution from infection to case confirmation is visualized in Fig. $1 \mathrm{~b}$.

\subsection{Data analysis}

All statistical analyses were performed in R (R Core Team, 2016). The non-negative least-square regression for the incidence estimations was implemented with the package 'CVXR' (Fu et al., 2020) and delay distributions were computed with the $\mathrm{R}$ package 'distr' (Ruckdeschel and Kohl, 2014).

\subsection{Data availability}

Data (measured RNA concentrations, flow rates and case numbers) are available at http://doi.org/10.5281/zenodo.4750572.

\section{Results and discussion}

\subsection{Method performance}

\subsubsection{PCR efficiency and limit of detection}

PCR efficiencies for all targets ranged from 94-111\% (Table S2). The $R^{2}$ of the pooled standard curves were $\geq 0.95$. No amplification signal was measured in the non-template and negative extraction controls confirming the absence of contamination during sample processing. The LOD corresponded to $4.2 \mathrm{gc} / \mathrm{ml}$ wastewater and $2.6 \mathrm{gc} / \mathrm{ml}$ wastewater for the SARS-CoV-2 $\mathrm{N} 1$ and $\mathrm{N} 2$ genes, respectively. The LODs reflect the difficulty of producing accurate calibration curves for SARS-CoV-2 in the low template range based on plasmid standards. This limitation, which was also reported by others (Chik et al., 2021; Gerrity et al., 2021) highlights the need for improved qPCR standards and more sensitive RT-qPCR assays to minimize variability and false negative results in SARS-CoV-2 RNA quantification. The LODs of the other targets are listed in Table S2.

\subsubsection{PCR inhibition}

Spiking RNA extracts with synthetic SARS-CoV-2 RNA reference material revealed minimal PCR inhibition on most samples. Specifically, with the exception of three samples from Lugano, all spiked RNA extracts exhibited N1 Cq values that fell within or only minimally beyond 1.5 cycles of the median Cq of a given WWTP (Figure S2).

\subsubsection{Reproducibility}

We compared quantifiable $\mathrm{N} 1$ concentrations determined in biological as well as in technical replicate samples (Figure S3). A good correlation $(\mathrm{r}=0.89)$ was obtained among biological replicates, indicating a high reproducibility of the overall processing pipeline. A good reproducibility was also found for technical replicates $(r=0.78)$.

\subsubsection{Recovery}

Three enveloped viruses - MHV, Sendai virus and $\Phi 6$ - were evaluated as SARS-CoV-2 surrogates to monitor virus recovery in our sample processing pipeline. As a member of the Coronaviridae family, MHV is the most similar to SARS-CoV-2 in terms of size (120 nm diameter) and genome structure (single-stranded RNA). Sendai virus has a single-stranded RNA genome, but is slightly larger in diameter than SARS-CoV-2 (150 nm). Besides SARS-CoV-2, this virus may also serve as a surrogate for viruses with pandemic potential in the Paramyxoviridae family, such as measles virus. The 


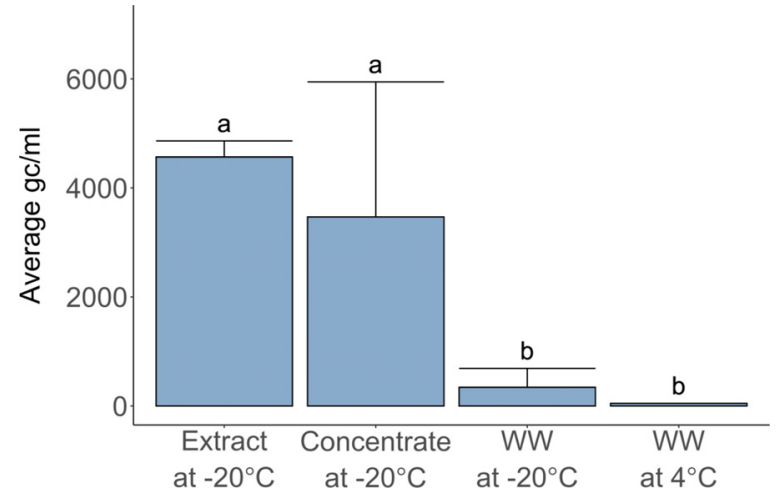

Fig. 2. Effect of sample storage over one month under different conditions on SARS$\mathrm{CoV}-2$ RNA concentrations (N1 gene target, gc/ml wastewater). Error bars represent standard deviations of replicate samples. Samples stored as non-processed raw wastewater (WW) at $4{ }^{\circ} \mathrm{C}$ or $-20{ }^{\circ} \mathrm{C}$ exhibited lower concentrations compared to samples stored at $-20{ }^{\circ} \mathrm{C}$ as concentrate (post ultrafiltration) or RNA extracts. Indices $\mathrm{a}$ and $\mathrm{b}$ denote experimental conditions yielding statistically different sample means.

novel RT-qPCR assay developed herein was able to quantify its concentration down to an LOD of $4.2 \mathrm{gc} / \mathrm{ml}$ (Table S2). Finally, $\Phi 6$ is the least similar surrogate to SARS-CoV-2. It has a smaller diameter $(85 \mathrm{~nm})$ and a different genome structure (double-stranded RNA).

In seven wastewater samples spiked with all three surrogate viruses, MHV and Sendai virus exhibited similar recoveries that mostly ranged from $0.1-1 \%$ (Figure S4). This range corresponds well to that reported by other groups using a similar processing pipeline (Pecson et al., 2021). If determined using $\Phi 6$, recoveries were 10 - to 100 -fold higher and more constant across samples. This confirms previous reports that recoveries depend strongly on the surrogate virus used (Pecson et al., 2021). Despite the better recovery of $\Phi 6$, we decided to utilize MHV or Sendai virus as recovery controls in this work, due to their higher structural similarity with SARS-CoV-2.

The recoveries in the samples from Lugano and Lausanne were determined using MHV (Figure S5). Recoveries were similar for both sites and mostly fell into the $0.1-1 \%$ range, with average values of $0.95 \%$ and $0.74 \%$ for Lugano and Lausanne, respectively. In the samples from Zurich, Sendai virus was used as the surrogate. Compared to Lugano and Lausanne, the recoveries were significantly lower, with an average of $0.17 \%$ (one-way ANOVA, $\mathrm{F}=6.82$, $\mathrm{p}<0.002$ ) (Figure S5). The lower recovery is unlikely to be a result of the use of Sendai virus, since MHV and Sendai virus yielded similar recoveries if assessed in the same sample (Figure S4). Instead, the lower recoveries in the Zurich samples may reflect the higher solids content in this WWTP. Enveloped viruses partition to wastewater solids (Ye et al., 2016), and hence a higher solids content leads to a reduced recovery of the surrogate virus from the liquid wastewater fraction.

\subsubsection{Fecal load}

The daily load of PMMoV was used as an indicator of the fecal load entering the WWTP. On average, the PMMoV loads corresponded to $8.9 \times 10^{15} \mathrm{gc} /$ day (Lugano), $3.1 \times 10^{16} \mathrm{gc} /$ day (Lausanne) and $1.8 \times 10^{16} \mathrm{gc} /$ day (Zurich) (Figure S6). In all but five samples the PMMoV load fell within the range of $5 \times 10^{15}$ to $5 \times 10^{16} \mathrm{gc} /$ day. The narrow range of the measured PMMoV loads further confirms the consistency of our virus concentration and extraction process.

\subsubsection{Effect of storage conditions on RNA stability}

As shown in Fig. 2, different storage procedures exert significantly different effects on SARS-CoV-2 RNA stability (one-way ANOVA, $\mathrm{F}=12.8, \mathrm{p}<0.001$ ). Storing raw wastewater at $4{ }^{\circ} \mathrm{C}$ or -
$20{ }^{\circ} \mathrm{C}$ for a month resulted in lower concentrations of SARS-CoV2 RNA compared to samples stored as concentrates or RNA extracts at $-20{ }^{\circ} \mathrm{C}$ (Tukey-Kramer, $\mathrm{p}<0.02$ ). The storage protocol used herein (raw wastewater at $-20^{\circ} \mathrm{C}$ ) had to be implemented before storage tests could be completed, and likely led to significant RNA decay. In future studies, wastewater samples should immediately be concentrated or extracted prior to storage.

\subsection{Longitudinal trends SARS-CoV-2 RNA loads and confirmed cases}

Concentrations of SARS-CoV-2 N1 and N2 gene targets in longitudinal samples are shown in Figure S7. The earliest detection of the N1 gene occurred in wastewater from Lugano on February 28, 2020, four days after the first COVID-19 case was observed in Switzerland. This confirms earlier reports that wastewater can serve as a sensitive indicator for virus circulation, even during periods of low disease prevalence (Ahmed et al., 2021; La Rosa et al., 2020; Medema et al., 2020; Randazzo et al., 2020).

$\mathrm{N} 1$ and $\mathrm{N} 2$ concentrations exhibited similar temporal trends, though N1 concentrations were on average 3-fold higher (Figure S7). Higher concentrations of the N1 gene were also reported by others (D'Aoust et al., 2021; Gerrity et al., 2021), though some studies have reported the N2 gene to yield higher results (Gonzalez et al., 2020; Medema et al., 2020). Given the superior quantification by $\mathrm{N} 1$ in this work, only this gene target was considered for all subsequent analyses.

We did not normalize N1 concentrations by fecal strength (PMMoV concentration) as suggested elsewhere (D'Aoust et al., 2021; Wu et al., 2020), because PMMoV concentrations were similar in all samples and were not correlated with N1 concentrations ( $\mathrm{r}=0.02-0.04)$ (Figure $\mathrm{S} 8$ ). We also did not correct $\mathrm{N} 1$ concentrations for recovery, because there was high inter-sample variation across the three surrogates tested, and it is uncertain which - if any - externally added surrogate accurately mimics the fate of SARS-CoV-2 during sample processing (Chik et al., 2021; Graham et al., 2021). Recovery values were strictly used for data quality control. Specifically, samples were excluded if two criteria were simultaneously met: the recovery of a sample was $>3 \times$ the median recovery for the site under consideration; and the concentrations measured by N1 and N2 differed by more than a factor 5 . This led to the exclusion of one biological replicate on three sampling days in Lugano (March 1819 and April 5), and both replicates for a single day in Lausanne (March 28).

Among the three WWTPs studied, Lausanne had the highest number of confirmed cases in its catchment (Fig. 3). Case numbers were similar in the catchments of the Lugano and Zurich WWTPs, even though Zurich's catchment encompasses approximately 3.6fold more inhabitants than Lugano's, and 1.9-fold more than Lausanne's. Consequently, the N1 concentrations in the Zurich WWTP were expected to be lower compared to Lugano and Lausanne, as confirmed by our measurements (Figure S7). To enable a direct comparison among WWTPs, we converted N1 concentrations into units of daily N1 load (Eq. 2). This unit accounts for differences in catchment size (via the daily wastewater flow rate), and also incorporates daily variability in the wastewater flow of a given WWTP.

Similar to data from other studies (Gerrity et al., 2021; Graham et al., 2021; Peccia et al., 2020), there was considerable day-to-day variability in both the N1 loads and the number of confirmed cases (Fig. 3). The variability in confirmed cases is increased by the fact that Switzerland reduces testing and reporting on weekends. To facilitate the visualization of pandemic trends in wastewater and case data, we therefore calculated weekly averages (Monday - Sunday) for each data set. The corresponding results are shown as solid lines in Fig. 3. As is evident, both data sets feature a prominent peak in late March. However, the wastewater peak 

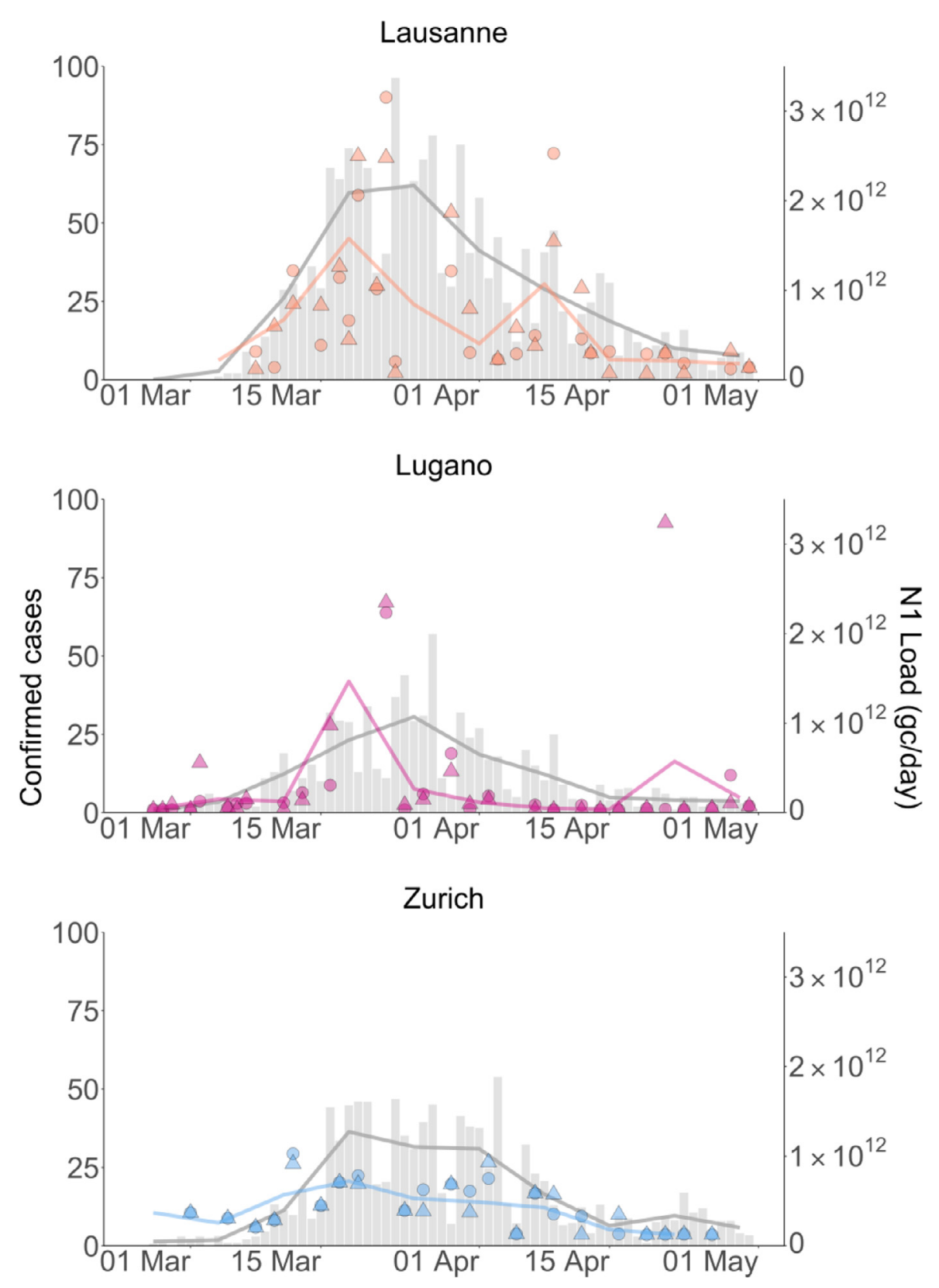

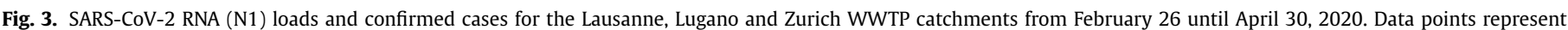

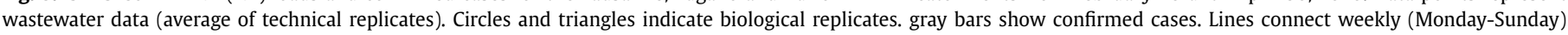
averages of SARS-CoV-2 RNA loads or confirmed cases.

shape is narrow, whereas the number of confirmed cases remained high for 2-3 weeks.

Despite the similarity in confirmed case numbers in the catchment, measured SARS-CoV-2 RNA loads were higher in the Lugano WWTP than in Zurich WWTP. There are a number of potential methodological explanations for this, including lower virus recovery in Zurich (Figure S5), reduced precision in quantifying low SARS-CoV-2 RNA copy numbers (Figure S7), and our sample storage protocol, which in retrospect was found to be non ideal (Fig. 2). The Zurich WWTP is located in the area of lowest disease prevalence and thus had the lowest starting concentrations of SARS-CoV-2 RNA among the WWTPs sampled. Further decay during storage of the Zurich samples may have lowered the concentrations below the LOD in all but the samples taken during the peak of the first wave.
3.3. Comparison of incidence dynamics from wastewater data, case numbers and SEIR models

To assess the ability to track disease dynamics with SARS-CoV2 loads in wastewater and confirmed cases, both data sets were used to estimate disease incidence over time (by deconvoluting the signals, see Materials and Methods). The resulting trends were compared to the reference incidence determined by an SEIR model (Lemaitre et al., 2020). While the SEIR model reports absolute infection numbers, this determination is currently not feasible for wastewater- or case number-derived estimates. For wastewater, estimating absolute infection numbers would require a better understanding of the magnitude of the shedding load $L$ (Eq. 4), the decay kinetics of SARS-CoV-2 RNA in the sewer system, and the true recovery of SARS-CoV-2 in our sample processing pipeline. 

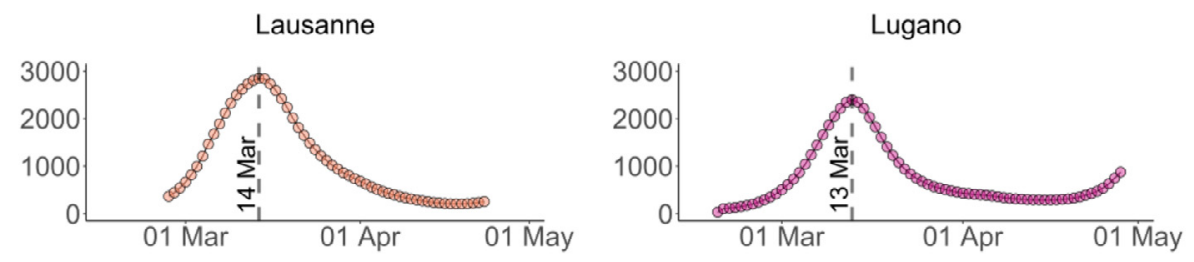

\section{Reference
(SEIR model)}
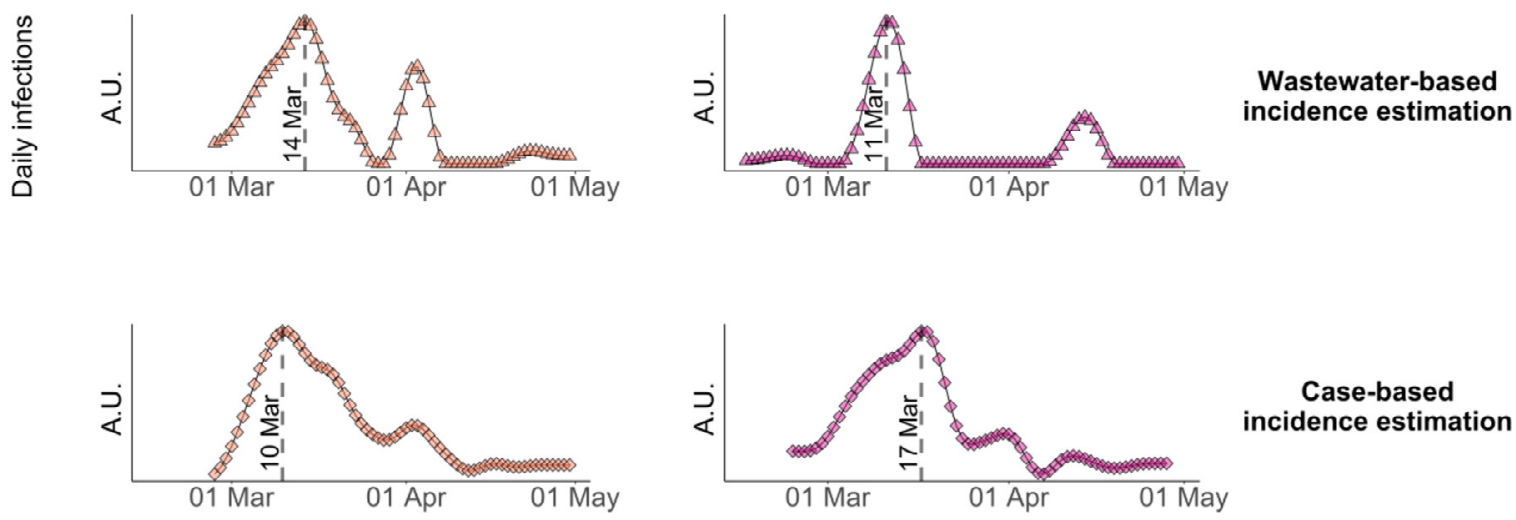

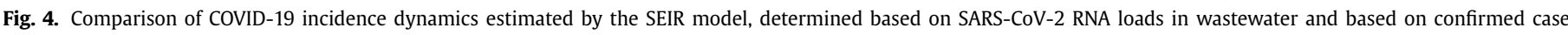

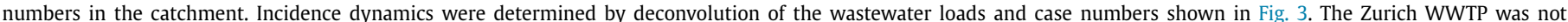
included in this analysis, because most wastewater samples yielded non-detectable SARS-CoV-2 RNA concentrations. A.U. = arbitrary units.

These parameters are currently not available, but may become better known in the future. For case numbers, the ratio of confirmed to total cases would have to be known, yet this parameter is associated with considerable uncertainty and variability during the first wave of the pandemic. We therefore only compared the incidence dynamics, but not the absolute incidence per day.

As shown in Fig. 4, both wastewater- and case number-derived incidence exhibited a pronounced peak in mid-March. In Lausanne, the wastewater-derived incidence exhibited the highest number of infections from March 13-15, which matches the peak of infections determined by the SEIR model. If estimated based on confirmed cases, the highest number of new infections occurred from March 9-11. Considering the delay distributions from infection to case confirmation (Fig. 1b), this time range mainly reflects cases observed from March 17-24, coinciding with Swisswide positivity rates $>10 \%$ (Figure $\mathrm{S} 1$ ). The premature timing of the peak may indicate that case numbers were truncated when testing capacity was exceeded and positivity rates were high.

In Lugano, wastewater-based incidence estimates yielded the highest infection numbers from March 10-12. This time frame partly overlapped with the SEIR-modelled infection peak, which occurred from March 12-14. In contrast, the incidence peak determined from confirmed cases appeared later (March 17-19) and differed in shape compared to the other incidence estimates. This is another indication testing capacity during this period was insufficient to capture the full extent of the rise in cases during the height of the first wave.

In both locations, the decline in new infections was better captured by case number- than by wastewater-based incidence estimates. In Lausanne, the case number-based incidence exhibited a slow decay in new infections from mid-March to late April, similar to the reference incidence dynamics. In contrast, the decay in the wastewater-based incidence was faster. In Lugano the case number-derived incidence was also able to capture the tail end of the wave, whereas new infections based on wastewater data rapidly dropped to the baseline.
Finally, the wastewater-based incidence dynamics in both locations exhibited a second, smaller peak in April, which was driven by few high load measurements in each location. In Lausanne this feature also appeared in the corresponding case number-derived incidence dynamics and thus reflects a local spike in infections. In contrast, the origin of the second peak in Lugano is not evident. It may stem from one or more shedders that are not permanent inhabitants of the Lugano WWTP catchment and were therefore not included in the catchment-specific case numbers (e.g., commuters or external patients hospitalized within the catchment). The April peaks were not apparent in the SEIR model, which may be explained by differences in the type of input data used to determine incidence dynamics. Whereas wastewater loads and case numbers were catchment-specific, the SEIR model was based on data for the entire canton. Local spikes in case numbers would thus appear attenuated in the reference incidence.

\section{Conclusions}

Our findings demonstrate that both confirmed case numbers and wastewater analysis are useful and independent metrics to estimate COVID-19 infection incidence dynamics. Wastewater outperformed case numbers with respect to the timing and shape of the peak incidence, whereas confirmed case numbers were a better indicator for incidence decline. In combination, the two metrics yielded complementary information on incidence dynamics that correspond well to the reference dynamics determined by compartmental models.

It is important to consider that all three approaches rely on a number of assumptions, all of which are associated with a degree of uncertainty. For example, the SEIR model is based exclusively on data pertaining to severe COVID-19 cases (hospitalizations, deaths), and may thus miss events among age classes that have a low severity rate but normal virus shedding. Wastewaterderived incidence dynamics suffer from uncertainties in the accuracy of the SLD. And cases-number derived estimates rely on the delay distribution between infection and case confirmation, which 
may vary with time and location. While the sources of uncertainties of these assumptions are conceptually understood, they remain difficult to quantify due to the lack of reference data. It is therefore important and encouraging that despite these uncertainties, comparable incidence dynamics were obtained with three independent approaches.

Differences in the incidence dynamics determined by wastewater and confirmed cases may ultimately also be exploited to inform on the duration and degree of clinical undertesting. To do so, however, both incidence estimates need to be further advanced. In future work, wastewater-derived estimates can be enhanced by increasing the wastewater sampling frequency to smooth out measurement outliers, developing more sensitive assays to quantify the viral RNA at low concentrations, better determining SARS-CoV-2 RNA recovery from wastewater, and establishing a representative shedding load profile. Case number-derived incidence estimates can be improved by taking into account variations in the delay distributions from symptom onset to case confirmation. In Switzerland, the mean delay varied from 3 to 8 days during the first wave (Huisman et al., 2020), yet herein it was held constant at 5.5 days.

Compared to the compartmental model, which relies on hospitalization and deaths, WBE can determine incidence dynamics with a faster turnaround time (RNA loads can be measured within 24 hours after sampling). Compared to clinical tests, an additional advantage of WBE is that a much lower number of samples is required to determine incidence dynamics with reasonable accuracy. During high positivity rate regimes, WBE can thus yield information on the trajectory of a pandemic that is potentially more precise, more readily available and more economical than information from clinical data. We contend that WBE should be included by epidemiologists and public health agencies as a useful pandemic monitoring tool during periods with high test positivity rates.

\section{Declaration of Competing Interest}

The authors declare that they have no known competing financial interests or personal relationships that could have appeared to influence the work reported in this paper.

\section{Acknowledgments}

This work was supported by the Swiss National Science Foundation (project 31CA30_196538), Eawag discretionary funds, and an EPFL COVID-19 grant. XFC was a fellow of the European Union's Horizon 2020 research and innovation program under the Marie Skłodowska-Curie Grant Agreement No. 754462. We thank the operators of the Lugano, Lausanne and Zurich WWTPs for providing samples, the Swiss Federal Office of Public Health for catchmentspecific case numbers, Jana Huisman for valuable input on SLDs, and Marie-Helene Corre, Elyse Stachler and Lea Caduff for lab assistance.

\section{Supplementary materials}

Supplementary material associated with this article can be found, in the online version, at doi:10.1016/j.watres.2021.117252.

\section{References}

Ahmed, W., Angel, N., Edson, J., Bibby, K., Bivins, A., O'Brien, J.W., Choi, P.M., Kitajima, M., Simpson, S.L., Li, J., Tscharke, B., Verhagen, R., Smith, W.J.M., Zaugg, J., Dierens, L., Hugenholtz, P., Thomas, K.V., Mueller, J.F., 2020. First confirmed detection of SARS-CoV-2 in untreated wastewater in Australia: A proof of concept for the wastewater surveillance of COVID-19 in the community. Sci. Total Environ. 728,138764 . doi:10.1016/j.scitotenv.2020.138764
Ahmed, W., Tscharke, B., Bertsch, P.M., Bibby, K., Bivins, A., Choi, P., Clarke, L., Dwyer, J., Edson, J., Nguyen, T.M.H., O’Brien, J.W., Simpson, S.L., Sherman, P., Thomas, K.V., Verhagen, R., Zaugg, J., Mueller, J.F., 2021. SARS-CoV-2 RNA monitoring in wastewater as a potential early warning system for COVID-19 transmission in the community: a temporal case study. Sci. Total Environ. 761, 144216. doi:10.1016/j.scitotenv.2020.144216.

Benefield, A.E., Skrip, L.A., Clement, A., Althouse, R.A., Chang, S., Althouse, B.M. 2020. SARS-CoV-2 Viral Load Peaks Prior to Symptom onset: a Systematic Review and Individual-Pooled Analysis of Coronavirus Viral Load from 66 Studies. medRxiv doi:10.1101/2020.09.28.20202028.

Besselsen, D.G., Wagner, A.M., Loganbill, J.K., 2002. Detection of rodent coronaviruses by use of fluorogenic reverse transcriptase-polymerase chain reaction analysis. Comp. Med. 52, 111-116.

Bi, Q., Wu, Yongsheng, Mei, S., Ye, C., Zou, X., Zhang, Z., Liu, X., Wei, L., Truelove, S.A., Zhang, T., Gao, W., Cheng, C., Tang, X., Wu, X., Wu, Yu, Sun, B., Huang, S., Sun, Y., Zhang, J., Ma, T., Lessler, J., Feng, T., 2020. Epidemiology and transmission of COVID-19 in 391 cases and 1286 of their close contacts in Shenzhen, China: a retrospective cohort study. Lancet Infect. Dis. 20, 911-919. doi:10.1016/S1473-3099(20)30287-5.

Bisseux, M., Debroas, D., Mirand, A., Archimbaud, C., Peigue-Lafeuille, H., Bailly, J.L., Henquell, C., 2020. Monitoring of enterovirus diversity in wastewater by ultra-deep sequencing: An effective complementary tool for clinical enterovirus surveillance. Water Res. 169, 115246. doi:10.1016/j.watres.2019.115246.

Chik, A.H.S., Glier M.B., Servos, M., Mangat, C.S., Pang, X-L., Oiu, Y. D'Aoust, P.M. Burnet, J.-B., Delatolla, R., Dorner, S., Geng, Q., Giesy Jr, J.P., McKay, R.M. Mulvey, M.R., Prystajecky, N., Srikanthan, N., Xie, Y., Conant, B., Hrudey, S.E., 2021. Comparison of approaches to quantify SARS-CoV-2 in wastewater using RT-qPCR: results and implications from a collaborative inter-laboratory study in Canada. J. Environ. Sci. (China) 107, 218-229.

Crits-Christoph, A., Kantor, R.S., Olm, M.R., Whitney, O.N., Al-Shayeb, B., Lou, Y.C., Flamholz, A., Kennedy, L.C., Greenwald, H., Hinkle, A., Hetzel, J., Spitzer, S. Koble, J., Tan, A., Hyde, F., Schroth, G., Kuersten, S., Banfield, J.F., Nelson, K.L. 2021. Genome Sequencing of Sewage Detects Regionally Prevalent SARS-CoV-2 Variants. MBio 12. doi:10.1128/mBio.02703-20.

D’Aoust, P.M., Mercier, E., Montpetit, D., Jia, J.-J., Alexandrov, I., Neault, N., Baig, A.T. Mayne, J., Zhang, X., Alain, T., Langlois, M.-A., Servos, M.R., MacKenzie, M., Figeys, D., MacKenzie, A.E., Graber, T.E., Delatolla, R., 2021. Quantitative analysis of SARS-CoV-2 RNA from wastewater solids in communities with low COVID19 incidence and prevalence. Water Res. 188, 116560. doi:10.1016/j.watres.2020. 116560

Fernandez-Cassi, X., Timoneda, N., Martínez-Puchol, S., Rusiñol, M., RodriguezManzano, J., Figuerola, N., Bofill-Mas, S., Abril, J.F., Girones, R., 2018. Metagenomics for the study of viruses in urban sewage as a tool for public health surveillance. Sci. Total Environ. 618, 870-880. doi:10.1016/j.scitotenv.2017.08. 249.

Fu, A., Narasimhan, B., Boyd, S., 2020. cvxr : an r package for disciplined convex optimization. J. Stat. Softw. 94. doi:10.18637/jss.v094.i14.

Gendron, L., Verreault, D., Veillette, M., Moineau, S., Duchaine, C., 2010. Evaluation of filters for the sampling and quantification of RNA phage aerosols. Aerosol Sci. Technol. 44, 893-901. doi:10.1080/02786826.2010.501351.

Gerrity, D., Papp, K., Stoker, M., Sims, A., Frehner, W., 2021. Early-pandemic wastewater surveillance of SARS-CoV-2 in Southern Nevada: Methodology, occurrence, and incidence/prevalence considerations. Water Res. X 10, 100086. doi:10.1016/ j.wroa.2020.100086.

Gonzalez, R., Curtis, K., Bivins, A., Bibby, K., Weir, M.H., Yetka, K., Thompson, H., Keeling, D., Mitchell, J., Gonzalez, D., 2020. COVID-19 surveillance in Southeastern Virginia using wastewater-based epidemiology. Water Res. 186, 116296. doi:10.1016/j.watres.2020.116296.

Graham, K.E., Loeb, S.K., Wolfe, M.K., Catoe, D., Sinnott-Armstrong, N., Kim, S., Yamahara, K.M., Sassoubre, L.M., Mendoza Grijalva, L.M., Roldan-Hernandez, L., Langenfeld, K., Wigginton, K.R., Boehm, A.B., 2021. SARS-CoV-2 RNA in Wastewater Settled Solids Is Associated with COVID-19 Cases in a Large Urban Sewershed. Environ. Sci. Technol. 55, 488-498, doi:10.1021/acs.est.0c06191.

Han, M.S., Seong, M.-W., Heo, E.Y., Park, J.H., Kim, N., Shin, S., Cho, S.I., Park, S.S., Choi, E.H., 2020. Sequential analysis of viral load in a neonate and her mother infected with severe acute respiratory syndrome coronavirus 2. Clin. Infect. Dis. 71, 2236-2239. doi:10.1093/cid/ciaa447.

Haramoto, E., Kitajima, M., Kishida, N., Konno, Y., Katayama, H., Asami, M., Akiba, M., 2013. Occurrence of pepper mild mottle virus in drinking water sources in Japan. Appl. Environ. Microbiol. 79, 7413-7418. doi:10.1128/AEM.02354-13.

Hovi, T., Shulman, L.M., van der Avoort, H., Deshpande, J., Roivainen, M., DE Gourville, E.M., 2012. Role of environmental poliovirus surveillance in global polio eradication and beyond. Epidemiol. Infect. 140, 1-13. doi:10.1017/ S095026881000316X.

Huisman, J.S., Scire, J., Angst, D.C., Neher, R.A., Bonhoeffer, S., Stadler, T., 2020. Estimation and Worldwide Monitoring of the Effective Reproductive Number of SARS-CoV-2. medRxiv 2020.11.26.20239368; doi: https://doi.org/10.1101/2020. 11.26.20239368.

Jahn, K., Dreifuss, D., Topolsky, I., Kull, A., Ganesanandamoorthy, P., FernandezCassi, X., Bänziger, C., Stachler, E., Fuhrmann, L., Jablonski, K.P., Chen, C., Aquino, C., Stadler, T., Ort, C., Kohn, T., Julian, T.R., Beerenwinkel, N., 2021. Detection of SARS-CoV-2 Variants in Switzerland by Genomic Analysis of Wastewater Samples. medRxiv doi:10.1101/2021.01.08.21249379. 
Kazama, S., Miura, T., Masago, Y., Konta, Y., Tohma, K., Manaka, T., Liu, X., Nakayama, D., Tanno, T., Saito, M., Oshitani, H., Omura, T., 2017. Environmental surveillance of norovirus genogroups I and II for sensitive detection of epidemic variants. Appl. Environ. Microbiol. 83. doi:10.1128/AEM.03406-16.

Kitajima, M., Iker, B.C., Pepper, I.L., Gerba, C.P., 2014. Relative abundance and treatment reduction of viruses during wastewater treatment processes-identification of potential viral indicators. Sci. Total Environ. 488-489, 290-296. doi:10.1016/j. scitotenv.2014.04.087.

La Rosa, G., Iaconelli, M., Mancini, P., Bonanno Ferraro, G., Veneri, C., Bonadonna, L., Lucentini, L., Suffredini, E., 2020. First detection of SARS-CoV-2 in untreated wastewaters in Italy. Sci. Total Environ. 736, 139652. doi:10.1016/j.scitotenv. 2020.139652.

Leibowitz, J., Kaufman, G., Liu, P., 2011. Coronaviruses: propagation, quantification, storage, and construction of recombinant mouse hepatitis virus. Curr. Protoc. Microbiol. Chapter 15. doi:10.1002/9780471729259.mc15e01s21, Unit 15E.1.

Lemaitre, J.C., Perez-Saez, J., Azman, A.S., Rinaldo, A., Fellay, J., 2020. Assessing the impact of non-pharmaceutical interventions on SARS-CoV-2 transmission in Switzerland. Swiss Med. Wkly. 150, w20295. doi:10.4414/smw.2020.20295.

Linton, N.M., Kobayashi, T., Yang, Y., Hayashi, K., Akhmetzhanov, A.R., Jung, S.-M., Yuan, B., Kinoshita, R., Nishiura, H., 2020. Incubation period and other epidemiological characteristics of 2019 novel coronavirus infections with right truncation: a statistical analysis of publicly available case data. J. Clin. Med. 9. doi:10.3390/jcm9020538.

Liu, P., Cai, J. Jia, R., Xia, S, Wang, X. Cao, L, Zeng, M., Xu, J, 2020. Dynamic surveillance of SARS-CoV-2 shedding and neutralizing antibody in children with COVID-19. Emerg. Microbes Infect. 9, 1254-1258. doi:10.1080/22221751.2020. 1772677.

Lu, X., Wang, L., Sakthivel, S.K., Whitaker, B., Murray, J., Kamili, S., Lynch, B., Malapati, L., Burke, S.A., Harcourt, J., Tamin, A., Thornburg, N.J., Villanueva, J.M., Lindstrom, S., 2020. US CDC real-time reverse transcription PCR panel for detection of severe acute respiratory syndrome coronavirus 2. Emerging Infect. Dis. 26. doi:10.3201/eid2608.201246.

Medema, G., Heijnen, L., Elsinga, G., Italiaander, R., Brouwer, A., 2020. Presence of SARS-Coronavirus-2 RNA in sewage and correlation with reported COVID-19 prevalence in the early stage of the epidemic in The Netherlands. Environ. Sci. Technol. Lett. doi:10.1021/acs.estlett.0c00357.

Merkes, C.M., Klymus K.E., Allison M.J., Goldberg C., Helbing C.C., Hunter M.E., Jackson C.A., Lance R.F., Mangan A.M., Monroe E.M., Piaggio A.J., Stokdyk J.P., Wilson C.C., Richter C. (2019) Generic qPCR Limit of Detection (LOD) /Limit of Quantification (LOQ) calculator. R Script. Available at: https://github.com/cmerkes/ qPCR_LOD_Calc. DOI: https://doi.org/10.5066/P9GT00GB.

Peccia, J., Zulli, A., Brackney, D.E., Grubaugh, N.D., Kaplan, E.H., CasanovasMassana, A., Ko, A.I., Malik, A.A., Wang, D., Wang, M., Warren, J.L., Weinberger, D.M., Arnold, W., Omer, S.B., 2020. Measurement of SARS-CoV-2 RNA in wastewater tracks community infection dynamics. Nat. Biotechnol. 38, 11641167. doi:10.1038/s41587-020-0684-z.
Pecson, B.M., Darby, E., Haas, C.N., Amha, Y.M., Bartolo, M., Danielson, R., Dearborn, Y., Di Giovanni, G., Ferguson, C., Fevig, S., Gaddis, E., Gray, D., Lukasik, G., Mull, B., Olivas, L., Olivieri, A., Qu, Y., 2021. Reproducibility and Sensitivity of 36 Methods to Quantify the SARS-CoV-2 Genetic Signal in Raw wastewater: Findings from an Interlaboratory Methods Evaluation in the U.S. Environ. Sci.: Water Res. Technol doi:10.1039/D0EW00946F.

R Core Team, 2016. R: A language and environment for statistical computing. $\mathrm{R}$ foundation for statistical computing, Vienna, Austria. https://www.R-project. org/.

Randazzo, W., Truchado, P., Cuevas-Ferrando, E., Simón, P., Allende, A., Sánchez, G., 2020. SARS-CoV-2 RNA in wastewater anticipated COVID-19 occurrence in a low prevalence area. Water Res. 181, 115942. doi:10.1016/j.watres.2020.115942.

Ruckdeschel, P., Kohl, M., 2014. General purpose convolution algorithm inS 4 classes by means of FFT. J. Stat. Softw. 59, 1-25. doi:10.18637/jss.v059.i04.

Sinclair, R.G., Choi, C.Y., Riley, M.R., Gerba, C.P., 2008. Pathogen surveillance through monitoring of sewer systems. Adv. Appl. Microbiol. 65, 249-269. doi:10.1016/ S0065-2164(08)00609-6.

Ståhlberg, A., Kubista, M., 2014. The workflow of single-cell expression profiling using quantitative real-time PCR. Expert Rev. Mol. Diagn. 14, 323-331. doi:10.1586/ 14737159.2014.901154.

Symonds, E.M., Nguyen, K.H., Harwood, V.J., Breitbart, M., 2018. Pepper mild mottle virus: a plant pathogen with a greater purpose in (waste)water treatment development and public health management. Water Res. 144, 1-12. doi:10.1016/j. watres.2018.06.066.

World Health Organization, 2020. Public Health Criteria to Adjust Public Health and Social Measures in the Context of COVID-19: Annex to Considerations in Adjusting Public Health and Social Measures in the Context of COVID-19, 12 May 2020. (No. WHO/2019-nCoV/Adjusting_PH_measures/Criteria/2020.1). World Health Organization.

Wu, F., Zhang, J., Xiao, A., Gu, X., Lee, W.L., Armas, F., Kauffman, K., Hanage, W., Matus, M., Ghaeli, N., Endo, N., Duvallet, C., Poyet, M., Moniz, K., Washburne, A.D. Erickson, T.B., Chai, P.R., Thompson, J., Alm, E.J., 2020. SARS-CoV-2 Titers in Wastewater Are Higher than Expected from Clinically Confirmed Cases. mSystems, p. 5. doi:10.1128/mSystems.00614-20.

Wu, Y., Guo, C., Tang, L., Hong, Z., Zhou, J., Dong, X., Yin, H., Xiao, Q., Tang, Y., Qu, X., Kuang, L., Fang, X., Mishra, N., Lu, J., Shan, H., Jiang, G., Huang, X., 2020. Prolonged presence of SARS-CoV-2 viral RNA in faecal samples. Lancet Gastroenterol. Hepatol. 5, 434-435. doi:10.1016/S2468-1253(20)30083-2.

Ye, Y., Ellenberg, R.M., Graham, K.E., Wigginton, K.R., 2016. Survivability, partitioning and recovery of enveloped viruses in untreated municipal wastewater. Environ. Sci. Technol. 50, 5077-5085. doi:10.1021/acs.est.6b00876.

Zhang, T., Breitbart, M., Lee, W.H., Run, J.-Q., Wei, C.L., Soh, S.W.L., Hibberd, M.L., Liu, E.T., Rohwer, F., Ruan, Y., 2006. RNA viral community in human feces: prevalence of plant pathogenic viruses. PLoS Biol. 4, e3. doi:10.1371/journal.pbio. 0040003. 